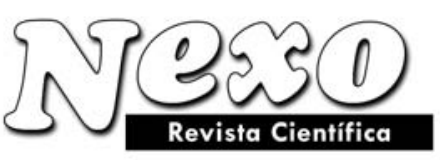

\title{
El producto de convolución de la derivada de orden de la delta de Dirac en un hipercono
}

\author{
Manuel A. Aguirre T. \\ Núcleo Consolidado de Matemática \\ Pura y Aplicada \\ Facultad de Ciencias Exactas \\ UNCentro,Pinto 399, \\ 7000 Tandil, \\ Argentina. \\ E-mail:maguirre@exa.unicen.edu.ar
}

(recibido/received: 14-Oct-2008; aceptado/accepted: 27-Marzo-2009)

\section{RESUMEN}

En este artículo se le da un sentido a los productos de convolución de $\delta^{(k)}\left(P_{+}\right) * \delta^{(l)}\left(P_{+}\right), \delta^{(k)}\left(P_{-}\right) * \delta^{(l)}\left(P_{-}\right)$, $\delta_{1}^{(k)}(P) * \delta_{1}^{(l)}(P) \quad$ y $\quad \delta_{2}^{(k)}(P) * \delta_{2}^{(l)}(P)$. En la primer sección, se le da un sentido a los productos $\delta^{(k)}\left(P_{+}\right) * \delta^{(l)}\left(P_{+}\right)$y $\delta^{(k)}\left(P_{-}\right) * \delta^{(l)}\left(P_{-}\right)$para $n$ impar y para el caso $n$ par hay que agregar la condiciones $k<\frac{n}{2}-1$ y $l<\frac{n}{2}-1$. En la segunda sección, se le da un sentido a los productos $\delta^{(k)}\left(P_{+}\right) * \delta^{(l)}\left(P_{+}\right)$, $\delta^{(k)}\left(P_{-}\right) * \delta^{(l)}\left(P_{-}\right), \delta_{1}^{(k)}(P) * \delta_{1}^{(l)}(P) \quad$ y $\quad \delta_{2}^{(k)}(P) * \delta_{2}^{(l)}(P) \quad$ bajo las condiciones $n \quad$ par, $\quad k \geq \frac{n}{2}-1 \quad$ y $l \geq \frac{n}{2}-1$.

Palabras clave: Teoría de distribuciones

AMS Clasificación de Temas: 46F10, 46F12

\section{ABSTRACT}

In this paper we give a sense to distributional convolution products of $\delta^{(k)}\left(P_{+}\right) * \delta^{(l)}\left(P_{+}\right), \delta^{(k)}\left(P_{-}\right) * \delta^{(l)}\left(P_{-}\right)$, $\delta_{1}^{(k)}(P) * \delta_{1}^{(l)}(P)$ and $\delta_{2}^{(k)}(P) * \delta_{2}^{(l)}(P)$. The first section, we give a sense to products of $\delta^{(k)}\left(P_{+}\right) * \delta^{(l)}\left(P_{+}\right)$ and $\delta^{(k)}\left(P_{-}\right) * \delta^{(l)}\left(P_{-}\right)$for odd $n$, as well as for even $n$ if $k<\frac{n}{2}-1$ and $l<\frac{n}{2}-1$. In the second section, we give a sense to products $\delta^{(k)}\left(P_{+}\right) * \delta^{(l)}\left(P_{+}\right), \quad \delta^{(k)}\left(P_{-}\right) * \delta^{(l)}\left(P_{-}\right), \delta_{1}^{(k)}(P) * \delta_{1}^{(l)}(P)$ and $\delta_{2}^{(k)}(P) * \delta_{2}^{(l)}(P)$ under conditions $n$ even, $k \geq \frac{n}{2}-1$ and $l \geq \frac{n}{2}-1$.

\footnotetext{
* Trabajo apoyado en parte por la Comisión de Investigaciones Científicas (C.I.C./Argentina).
} 
A. Aguirre

Keywords: Theory of Distributions

AMS Subject Classification: 46F10, 46F12 


\section{A. Aguirre}

\section{Introducción.}

Sea $x=\left(x_{1}, x_{2}, \ldots, x_{n}\right)$ un punto en el espacio $n$-dimensional Euclideano $R^{n}$.

Consideremos una forma cuadrática en $n$ variables definida por

$$
P=P(x)=x_{1}^{2}+\cdots x_{p}^{2}-x_{p+1}^{2} \cdots-x_{p+q}^{2}
$$

donde $p+q=n$ es la dimensión del espacio.

Llamamos $C^{\infty}$ al espacio $\varphi(x)$ de funciones infinitamente derivables y con soporte compacto definidas de $R^{n}$ en $R$.

De ([1]), página 253, fórmula (2)), la distribución $P_{+}^{\lambda}$ es definida por

$$
\left(P_{+}^{\lambda}, \varphi\right)=\int_{P>0}(P(x))^{\lambda} \varphi(x) d x
$$

donde $\lambda$ es un número complejo y $d x=d x_{1} d x_{2} \ldots d x_{n}$. Para $\operatorname{Real}(\lambda) \geq 0$, esta integral converge y es una función analítica de $\lambda$. La prolongación analítica a la parte Real $(\lambda)<0$ puede ser usada para extender la definición de $\left(P_{+}^{\lambda}, \varphi\right)$. Más aún de ([Gelfand], página 254), se tiene,

$$
\left(P_{+}^{\lambda}, \varphi\right)=\int_{0}^{\infty} u^{\lambda+\frac{p+q}{2}-1}{ }_{q} \Phi_{\lambda}(u) d u
$$

donde

$$
\begin{gathered}
{ }_{q} \Phi_{\lambda}(u)=\frac{1}{4} \int_{0}^{\infty} t^{\frac{q-2}{2}}(1-t)^{\lambda} \phi_{1}(u, t u) d t \\
\phi(r, s)=\phi_{1}(u, v) \\
\phi(r, s)=\int \varphi d \Omega_{p} d \Omega_{q} \\
r=\sqrt[2]{x_{1}^{2}+\ldots+x_{p}^{2}} \text { and } s=\sqrt[2]{x_{p+1}^{2}+\ldots+x_{p+q}^{2}}
\end{gathered}
$$

Aquí, $d \Omega_{p}$ y $d \Omega_{q}$ son los elementos de áreas de la superficie en la esfera unitaria en $R^{p}$ y $R^{q}$ respectivamente.

En forma similar podemos también definir la función generalizada $P_{-}^{\lambda}$ por

$$
\left(P_{-}^{\lambda}, \varphi\right)=\int_{-P>0}(-P(x))^{\lambda} \varphi(x) d x
$$

Más aún se obtiene

$$
\left(P_{-}^{\lambda}, \varphi\right)=\int_{0}^{\infty} v^{\lambda+\frac{p+q}{2}-1} \Phi_{\lambda}(v) d v
$$


donde

$$
{ }_{p} \Phi_{\lambda}(u)=\frac{1}{4} \int_{0}^{\infty} t^{\frac{p-2}{2}}(1-t)^{\lambda} \phi_{1}(v t, v) d t
$$

De (1) la ecuación de la hipersuperficie $P=0$ define un hipercono con un punto singular (el vértice) en el origen.

Por otro lado, de ([1], página 249), se tiene,

$$
\left(\delta^{(k)}(P), \varphi\right)=\int_{0}^{\infty}\left[\left(\frac{\partial}{2 s \partial s}\right)^{k}\left\{s^{q-2} \frac{\phi(r, s)}{2}\right\}\right]_{s=r} r^{p-1} d r
$$

y

$$
\left(\delta^{(k)}(P), \varphi\right)=(-1)^{k} \int_{0}^{\infty}\left[\left(\frac{\partial}{2 r \partial r}\right)^{k}\left\{r^{p-2} \frac{\phi(r, s)}{2}\right\}\right]_{r=s} s^{q-1} d s
$$

donde $\phi(r, s)$ es definida por la ecuación (6).

También de ([1], página 250), las funciones generalizadas $\delta_{1}^{(k)}(P)_{\text {y }} \delta_{2}^{(k)}(P)$ están definidas por

$$
\left(\delta_{1}^{(k)}(P), \varphi\right)=\int_{0}^{\infty}\left[\left(\frac{\partial}{2 s \partial s}\right)^{k}\left\{s^{q-2} \frac{\phi(r, s)}{2}\right\}\right]_{s=r} r^{p-1} d r
$$

$\mathrm{y}$

$$
\left(\delta_{2}^{(k)}(P), \varphi\right)=(-1)^{k} \int_{0}^{\infty}\left[\left(\frac{\partial}{2 r \partial r}\right)^{k}\left\{r^{p-2} \frac{\phi(r, s)}{2}\right\}\right]_{r=s} s^{q-1} d s
$$

donde $\phi(r, s)$ es $r^{1-p} s^{1-q}$ multiplicada por la integral de $\varphi$ sobre la superficie $x_{1}^{2}+x_{2}^{2}+\cdots+x_{p}^{2}=r^{2}$ y $x_{p+1}^{2}+\cdots+x_{p+q}^{2}=s^{2}$.

Las integrales convergen y coinciden para

$$
k<\frac{p+q-2}{2} .
$$

Si, por otra parte,

$$
k \geq \frac{p+q-2}{2}
$$

esas integrales deberían entenderse en el sentido de la regularización (ver [1], página 250 ).

Ahora en general $\delta_{1}^{(k)}(P)$ y $\delta_{2}^{(k)}(P)$ pueden no ser la misma función generalizada. Note que la definición de estas funciones generalizadas implica que en cualquier caso

$$
\delta_{2}^{(k)}(P)=(-1)^{k} \delta_{1}^{(k)}(-P) .
$$

De ([1]), página 278), las siguientes fórmulas son válidas,

$$
\delta^{(k)}\left(P_{+}\right)=(-1)^{k} k ! \operatorname{Re} s_{\lambda=-k-1} P_{+}^{\lambda}
$$

y 


$$
\delta^{(k)}\left(P_{-}\right)=(-1)^{k} k ! \operatorname{Re} s_{\lambda=-k-1} P_{-}^{\lambda}
$$

Por otra parte, de ([1] página 278), para $n$ impar y para $n$ par pero bajo la condición $k<\frac{n}{2}-1$ se tiene,

$$
\delta^{(k)}\left(P_{+}\right)=\delta_{1}^{(k)}(P)=\delta^{(k)}(P)
$$

$\mathrm{y}$

$$
\delta^{(k)}\left(P_{-}\right)=\delta_{1}^{(k)}(-P)
$$

Mientras que en el caso en que la dimensión $n$ sea par y $k \geq \frac{n}{2}-1$

$$
\delta^{(k)}\left(P_{+}\right)-\delta_{1}^{(k)}(P)
$$

$\mathrm{y}$

$$
\delta^{(k)}\left(P_{-}\right)-\delta_{1}^{(k)}(-P)
$$

son funciones generalizadas concentradas en el vértice del cono $P=0$ ([1], página 279). De([1], página 279), se tiene:

Si $p$ y $q$ son pares y si $k \geq \frac{n}{2}-1$, entonces

$$
(-1)^{k} \delta^{(k)}\left(P_{+}\right)-\delta^{(k)}\left(P_{-}\right)=a_{q, n, k} L^{k+1-\frac{n}{2}}\{\delta(x)\}
$$

mientras que en todos los otros casos

$$
\delta^{(k)}\left(P_{-}\right)=(-1)^{k} \delta^{(k)}\left(P_{+}\right) .
$$

En (25)

$$
a_{q, n, k}=\frac{(-1)^{\frac{q}{2}} \pi^{\frac{n}{2}}}{4^{k-\frac{n}{2}+1}\left(k-\frac{n}{2}+1\right) !}
$$

y $L^{j}$ es el operador diferencial lineal homogéneo iterado $j$ veces,

$$
L^{j}=\left\{\frac{\partial^{2}}{\partial x_{1}^{2}}+\cdots+\frac{\partial^{2}}{\partial x_{p}^{2}}-\frac{\partial^{2}}{\partial x_{p+1}^{2}}-\cdots-\frac{\partial^{2}}{\partial x_{p+q}^{2}}\right\}^{j}
$$

es decir, es el operador ultrahiperbólico $L=\left\{\frac{\partial^{2}}{\partial x_{1}^{2}}+\cdots+\frac{\partial^{2}}{\partial x_{p}^{2}}-\frac{\partial^{2}}{\partial x_{p+1}^{2}}-\cdots-\frac{\partial^{2}}{\partial x_{p+q}^{2}}\right\}$ iterado $j$ veces.

De([1], página 255), $\left(P_{+}^{\lambda}, \varphi\right)$ tiene dos conjuntos de singularidades a saber:

$$
\lambda=-1,-2,-3, \ldots
$$




$$
\lambda=-\frac{n}{2},-\frac{n}{2}-1, \ldots
$$

y de ([1], páginas 256-269 y página 352), se tiene,

$$
\begin{gathered}
\operatorname{Re} s_{\lambda=-k-1} P_{+}^{\lambda}=\frac{(-1)^{k}}{k !} \delta_{1}^{(k)}(P) \text { si } p \text { es par y } q \text { es impar } \\
\operatorname{Re} s_{\lambda=-k-1} P_{+}^{\lambda}=\frac{(-1)^{k}}{k !} \delta_{1}^{(k)}(P) \text { si } p \text { es impar y } q \text { es par, } \\
\operatorname{Re} s_{\lambda=-\frac{n}{2}-k} P_{+}^{\lambda}=0 \text { si } p \text { es par y } q \text { es impar }
\end{gathered}
$$

$\mathrm{y}$

$$
\operatorname{Re} s_{\lambda=-\frac{n}{2}-k} P_{+}^{\lambda}=\frac{(-1)^{\frac{q}{2}} \pi^{\frac{n}{2}}}{4^{k} k ! \Gamma\left(\frac{n}{2}+k\right)} L^{k}\{\delta(x\} \text { si } p \text { es impar y } q \text { es par }
$$

Donde $L^{k}$ es el operador ultra hiperbólico iterado $k$-veces definido por medio de la fórmula (28). En forma similar $\left(P_{-}^{\lambda}, \varphi\right)$ tiene singularidades en los mismos puntos que $\left(P_{+}^{\lambda}, \varphi\right)$ y tomando en cuenta que todo lo que se dice acerca de $P_{+}^{\lambda}$ también vale para $P_{-}^{\lambda}$ excepto que $p_{\text {y }} q$ debe ser intercambiados, y en todas las fórmulas que corresponde a $\delta_{1}^{(k)}(P)$ deben ser reemplazadas por

$$
\delta_{1}^{(k)}(-P)=(-1)^{k} \delta_{2}^{(k)}(P)
$$

y $(L)$ por $(-L)$ (ver [1], páginas 279 y 352 ), luego se tiene,

$$
\begin{gathered}
\operatorname{Re}_{\lambda=-k-1} P_{-}^{\lambda}=\frac{(-1)^{k}}{k !} \delta_{1}^{(k)}(-P) \text { si } p \text { es impar y } q \text { par } \\
\operatorname{Re} s_{\lambda=-k-1} P_{-}^{\lambda}=\frac{(-1)^{k}}{k !} \delta_{1}^{(k)}(-P) \text { si } p \text { es par y } q \text { impar } \\
\operatorname{Re}_{\lambda=-\frac{n}{2}-k} P_{-}^{\lambda}=0 \text { si } p \text { es impar y } q \text { par }
\end{gathered}
$$

$\mathrm{y}$

$$
\operatorname{Re} s_{\lambda=-\frac{n}{2}-k} P_{-}^{\lambda}=\frac{(-1)^{\frac{p}{2}} \pi^{\frac{n}{2}}}{4^{k} k ! \Gamma\left(\frac{n}{2}+k\right)}(-L)^{k}\{\delta(x\} \text { si } p \text { es par y } q \text { impar. }
$$

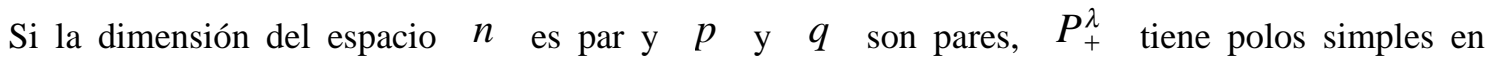
$\lambda=-\frac{n}{2}-k$, donde $k$ es un entero no-negativo, y el residuo es dado por la siguiente fórmula

$$
\begin{gathered}
\operatorname{Re}_{\lambda=-\frac{n}{2}-k, k=0,1,2, . .} P_{+}^{\lambda}=\frac{(-1)^{\frac{n}{2}+k-1}}{\Gamma\left(\frac{n}{2}+k\right)} \delta_{1}^{\left(\frac{n}{2}+k-1\right)}(P)+ \\
+\frac{(-1)^{\frac{q}{2}} \pi^{\frac{n}{2}}}{4^{k} k ! \Gamma\left(\frac{n}{2}+k\right)} L^{k}\{\delta(x\}
\end{gathered}
$$

([1], página 268), donde $L^{k}$ es definido por (28).

Si, por otra parte, $p$ y $q$ son impares, $P_{+}^{\lambda}$ tiene polo de orden 2 en $\lambda=-\frac{n}{2}-k$ y de ([1], página 269), se tiene 


$$
\begin{aligned}
& \operatorname{Re}_{\lambda=-\frac{n}{2}-k} P_{+}^{\lambda}=\frac{(-1)^{\frac{n}{2}+k-1}}{\Gamma\left(\frac{n}{2}+k\right)} \delta_{1}^{\left(\frac{n}{2}+k-1\right)}(P)+ \\
& +\frac{(-)^{\frac{q+1}{2}} \pi^{\frac{n}{2}-1}}{2^{2 k} k ! \Gamma\left(\frac{n}{2}+k\right)}\left[\psi\left(\frac{p}{2}\right)-\psi\left(\frac{n}{2}\right)\right] L^{k}\{\delta(x)\}
\end{aligned}
$$

donde

$$
\psi(x)=\frac{\Gamma^{\prime}(x)}{\Gamma(x)}
$$

y $\Gamma(x)$ es la función gama definida por

$$
\Gamma(x)=\int_{0}^{\infty} e^{-z} z^{x-1} d z
$$

Para valores enteros y valores fraccionarios la función $\psi(x)$ está dada por:

$$
\begin{gathered}
\psi(k)=-\gamma+1+\frac{1}{2}+\cdots+\frac{1}{k-1} \\
\psi\left(k+\frac{1}{2}\right)=-\gamma-2 \ln (2)+2\left(1+\frac{1}{3}+\cdots+\frac{1}{2 k-1}\right)
\end{gathered}
$$

donde $\gamma$ es la constante de Euler.

En forma similar

$$
\begin{gathered}
\operatorname{Re} s_{\lambda=-\frac{n}{2}-k} P_{-}^{\lambda}=\frac{(-1)^{\frac{n}{2}+k-1}}{\Gamma\left(\frac{n}{2}+k\right)} \delta_{1}^{\left(\frac{n}{2}+k-1\right)}(-P)+ \\
+\frac{(-1)^{\frac{p}{2}} \pi^{\frac{n}{2}}}{4^{k} k ! \Gamma\left(\frac{n}{2}+k\right)}(-L)^{k}\{\delta(x\} \operatorname{si} p \text { y } q \text { son impares. }
\end{gathered}
$$

$\mathrm{y}$

$$
\begin{gathered}
\operatorname{Re} s_{\lambda=-\frac{n}{2}-k} P_{-}^{\lambda}=\frac{(-1)^{\frac{n}{2}+k-1}}{\Gamma\left(\frac{n}{2}+k\right)} \delta_{1}^{\left(\frac{n}{2}+k-1\right)}(-P)+ \\
+\frac{(-)^{\frac{p+1}{2}} \pi^{\frac{n}{2}-1}}{2^{2 k} k ! \Gamma\left(\frac{n}{2}+k\right)} \\
{\left[\psi\left(\frac{q}{2}\right)-\psi\left(\frac{n}{2}\right)\right](-L)^{k}\{\delta(x)\} \text { si } p \text { y } q \text { son impar. }}
\end{gathered}
$$

En este artículo se le da un sentido a los siguientes productos distribucionales: 


$$
\begin{gathered}
\delta^{(k)}\left(P_{+}\right) * \delta^{(l)}\left(P_{+}\right) \\
\delta^{(k)}\left(P_{-}\right) * \delta^{(l)}\left(P_{-}\right) \\
\delta_{1}^{(k)}(P) * \delta_{1}^{(l)}(P)
\end{gathered}
$$

$\mathrm{y}$

$$
\delta_{2}^{(k)}(P) * \delta_{2}^{(l)}(P)
$$

Los resultados se dividen en dos secciones. En la primera sección se le da un sentido a los siguientes productos de convolución:

$$
\delta^{(k)}\left(P_{+}\right) * \delta^{(l)}\left(P_{+}\right)
$$

$\mathrm{y}$

$$
\delta^{(k)}\left(P_{-}\right) * \delta^{(l)}\left(P_{-}\right)
$$

para $n$ impar, y para $n$ par si $k<\frac{n}{2}-1$.

En la segunda sección se le da un sentido a los productos distribucionales

$$
\begin{gathered}
\delta^{(k)}\left(P_{+}\right) * \delta^{(l)}\left(P_{+}\right) \\
\delta^{(k)}\left(P_{-}\right) * \delta^{(k)}\left(P_{-}\right) \\
\delta_{1}^{(k)}(P) * \delta_{1}^{(l)}(P)
\end{gathered}
$$

$\mathrm{y}$

$$
\delta_{2}^{(k)}(P) * \delta_{2}^{(l)}(P)
$$

bajo las condiciones $n$ par, $k \geq \frac{n}{2}-1_{\text {y }} l \geq \frac{n}{2}-1$.

2. Los productos de la convolución $\delta^{(k)}\left(P_{+}\right) * \delta^{(l)}\left(P_{+}\right)$y $\quad \delta^{(k)}\left(P_{-}\right) * \delta^{(l)}\left(P_{-}\right)$. Para obtener dichos productos de convolución se necesitan las siguientes fórmulas:

$$
\left\{\frac{P_{-}^{\lambda}}{\Gamma(\lambda+1)}\right\}^{\wedge}=-a_{\lambda, n . i} e^{-\frac{q \pi i}{2}}(Q-i 0)^{-\lambda-\frac{n}{2}}-e^{\frac{q \pi i}{2}}(Q+i 0)^{-\lambda-\frac{n}{2}}
$$

([1], página 284 fórmula 4) y

$$
\left\{\frac{P_{+}^{\lambda}}{\Gamma(\lambda+1)}\right\}^{\wedge}=a_{\lambda, n . i} e^{-\left(\lambda+\frac{q}{2}\right) \pi i}(Q-i 0)^{-\lambda-\frac{n}{2}}-e^{\left(\lambda+\frac{q}{2}\right) \pi i}(Q+i 0)^{-\lambda-\frac{n}{2}}
$$

([1], página 284, fórmula 4), donde 


$$
(Q \pm i o)^{\lambda}=\lim _{\varepsilon \rightarrow 0}\left(Q \pm i \varepsilon|x|^{2}\right)^{\lambda}
$$

([1], página 275),

$$
Q=Q(y)=y_{1}^{2}+\cdots+y_{p}^{2}-y_{p+1}^{2}-\cdots-y_{p+q}^{2}
$$

$p+q=n$ dimensión del espacio, $\varepsilon$ es un número real tal que $\varepsilon>0$, el símbolo $\wedge$ indica transformada de Fourier,

$$
\{f\}^{\wedge}=\int e^{-\langle x, y\rangle} f(x) d x
$$

$\mathrm{y}$

$$
a_{\lambda, n . i}=2^{2 \lambda+n} \pi^{\frac{n}{2}-1} \Gamma\left(\lambda+\frac{n}{2}\right)(2 i)^{-1} .
$$

Usando las fórmulas

$$
(P \pm i 0)^{\lambda}=P_{+}^{\lambda}+e^{ \pm \lambda \pi i} P_{-}^{\lambda}
$$

([1], página 76),

$$
\begin{gathered}
P_{+}^{\lambda}=(-2 i \operatorname{sen} \lambda \pi)^{-1}\left(e^{-\lambda \pi i}(P+i 0)^{\lambda}-e^{\lambda \pi i}(P-i 0)^{\lambda}\right) \\
P_{-}^{\lambda}=(2 i \operatorname{sen} \lambda \pi)^{-1}\left((P+i 0)^{\lambda}-(P-i 0)^{\lambda}\right)
\end{gathered}
$$

$\mathrm{y}$

$$
\Gamma(z) \Gamma(1-z)=\frac{\pi}{\operatorname{sen} \lambda \pi}
$$

([8], página 344).

De(49) and (50) se tiene,

$$
\left\{\frac{P_{-}^{\lambda}}{\Gamma(\lambda+1)}\right\}^{\wedge}=2^{2 \lambda+n} \pi^{\frac{n}{2}-1}(-1)^{\frac{q-1}{2}}\left[Q_{+}^{-\lambda-\frac{n}{2}}+\cos \left(\lambda+\frac{n}{2}\right) Q_{-}^{-\lambda-\frac{n}{2}}\right]
$$

si $q$ es impar y $p$ es par ( $n$ impar),

$$
\left\{\frac{P_{+}^{\lambda}}{\Gamma(\lambda+1)}\right\}^{\wedge}=-2^{2 \lambda+n} \pi^{\frac{n}{2}-1}(-1)^{\frac{q-1}{2}}\left[Q_{+}^{-\lambda-\frac{n}{2}}+\cos \left(\lambda+\frac{n}{2}\right) Q_{-}^{-\lambda-\frac{n}{2}}\right]
$$

si $q$ es impary $p$ es par ( $n$ impar),

$$
\left\{\frac{P_{-}^{\lambda}}{\Gamma(\lambda+1)}\right\}^{\wedge}=-2^{2 \lambda+n} \pi^{\frac{n}{2}}(-1)^{\frac{q}{2}} \frac{Q_{-}^{-\lambda-\frac{n}{2}}}{\Gamma\left(-\lambda-\frac{n}{2}+1\right)}
$$

si $p$ y $q$ son pares, 


$$
\left\{\frac{P_{+}^{\lambda}}{\Gamma(\lambda+1)}\right\}^{\wedge}=-2^{2 \lambda+n} \pi^{\frac{n}{2}}(-1)^{\frac{p}{2}} \frac{Q^{-\lambda-\frac{n}{2}}}{\Gamma\left(-\lambda-\frac{n}{2}+1\right)}
$$

si $p$ y $q$ son pares,

$$
\left\{\frac{P_{-}^{\lambda}}{\Gamma(\lambda+1)}\right\}^{\wedge}=2^{2 \lambda+n} \pi^{\frac{n}{2}-1} 2^{-1}(-1)^{\frac{q-1}{2}}\left[(Q-i 0)^{-\lambda-\frac{n}{2}}+(Q+i 0)^{-\lambda-\frac{n}{2}}\right]
$$

si $p$ y $q$ son impares, $\mathrm{y}$

$$
\left\{\frac{P_{+}^{\lambda}}{\Gamma(\lambda+1)}\right\}^{\wedge}=-2^{2 \lambda+n} \pi^{\frac{n}{2}-1} 2^{-1}(-1)^{\frac{q-1}{2}}\left[e^{-\lambda \pi i}(Q-i 0)^{-\lambda-\frac{n}{2}}+e^{\lambda \pi i}(Q+i 0)^{-\lambda-\frac{n}{2}}\right]
$$

si $p$ y $q$ son impares.

De (56), (57), (58), (59) y usando las propiedades

$$
\left\{\lim _{\lambda \rightarrow-k} \frac{P_{+}^{\lambda}}{\Gamma(\lambda+1)}\right\}^{\wedge}=\lim _{\lambda \rightarrow-k}\left\{\frac{P_{+}^{\lambda}}{\Gamma(\lambda+1)}\right\}^{\wedge}
$$

([4], página 125, fórmula (98)) y la fórmula (20) se obtiene la siguiente fórmula

$$
\left\{\delta^{(k)}\left(P_{-}\right)\right\}^{\wedge}=-\frac{(-1)^{k} 2^{n-2(k+1)} \pi^{\frac{n}{2}}(-1)^{\frac{p}{2}}}{\Gamma\left(2-\frac{n}{2}+k\right)} Q_{+}^{k+1-\frac{n}{2}}
$$

si $q$ es impar y $p$ es par ( $n$ impar ),

$$
\left\{\delta^{(k)}\left(P_{+}\right)\right\}^{\wedge}=\frac{(-1)^{k} 2^{n-2(k+1)} \pi^{\frac{n}{2}}(-1)^{\frac{p}{2}}}{\Gamma\left(2-\frac{n}{2}+k\right)} Q_{+}^{k+1-\frac{n}{2}}
$$

si $p$ es par y $q$ es impar ( $n$ impar),

$$
\left\{\delta^{(k)}\left(P_{-}\right)\right\}^{\wedge}=-2^{n-2(k+1)} \pi^{\frac{n}{2}}(-1)^{\frac{q}{2}} \delta^{\left(\frac{n}{2}-k-1\right)}\left(Q_{-}\right)
$$

si $p_{\text {y }} q$ son pares y $k<\frac{n}{2}-1$, y

$$
\left\{\delta^{(k)}\left(P_{+}\right)\right\}^{\wedge}=-2^{n-2(k+1)} \pi^{\frac{n}{2}}(-1)^{\frac{p}{2}} \delta^{\left(\frac{n}{2}-k-1\right)}\left(Q_{+}\right)
$$

si $p$ y $q$ son pares $\mathrm{y} k<\frac{n}{2}-1$.

Cuando $p$ y $q$ son impares de (60) y (61) y usando la fórmula

$$
(Q \pm i 0)^{-k-1}=p f Q^{-k-1} \mp \frac{\pi i(-1)^{k}}{k !} \delta^{(k)}(Q)
$$

([9], página 577) la cual es válida si $k \neq \frac{n}{2}+s-1, s=1,2, \ldots$, donde $p f Q^{-k-1}$ significa la parte finita de $Q^{\lambda}$ en $\lambda=-k-1$ ([1],página 86) se tiene, 


$$
\left\{\delta^{(k)}\left(P_{-}\right)\right\}^{\wedge}=2^{n-2(k+1)} 2^{-1} \pi^{\frac{n}{2}}(-1)^{\frac{q-1}{2}} \Gamma\left(\frac{n}{2}-k-1\right) p f Q^{k+1-\frac{n}{2}}
$$

si $p$ y $q$ son impares y $k<\frac{n}{2}-1$, y

$$
\left\{\delta^{(k)}\left(P_{+}\right)\right\}^{\wedge}=(-1)^{k} 2^{n-2(k+1)} 2^{-1} \pi^{\frac{n}{2}}(-1)^{\frac{q-1}{2}} \Gamma\left(\frac{n}{2}-k-1\right) p f Q^{k+1-\frac{n}{2}}
$$

si $p_{\text {y }} q$ son impares y $k<\frac{n}{2}-1$.

Por otro parte, usando que $\delta^{(k)}\left(P_{ \pm}\right)$es una distribución de clase $S^{\prime}$ ([5], página 142) donde $S^{\prime}$ es el dual de $S$ y $S$ es el espacio de Schwartz ([7], página 233) y considerando el teorema clásico de Schwartz ([Schwartz], página 268, fórmula(IV,8,4) la siguiente fórmula es válida

$$
\left\{\delta^{(k)}\left(P_{+}\right) * \delta^{(l)}\left(P_{+}\right)\right\}^{\wedge}=\left\{\delta^{(k)}\left(P_{+}\right)\right\}^{\wedge} \cdot\left\{\delta^{(l)}\left(P_{+}\right)\right\}^{\wedge}
$$

$\mathrm{y}$

$$
\left\{\delta^{(k)}\left(P_{-}\right) * \delta^{(l)}\left(P_{-}\right)\right\}^{\wedge}=\left\{\delta^{(k)}\left(P_{-}\right)\right\}^{\wedge} \cdot\left\{\delta^{(l)}\left(P_{-}\right)\right\}^{\wedge}
$$

De (63), (64), (65), (66) y usando la propiedad

$$
P_{ \pm}^{\lambda} \cdot P_{ \pm}^{\mu}=P_{ \pm}^{\lambda+\mu}
$$

([6], página 39) donde $\lambda$ y $\mu$ son números complejos tales que $\lambda, \mu, \lambda+\mu \neq-\frac{n}{2}-t$, $t=0,1, \ldots$ y $\lambda, \mu, \lambda+\mu \neq-1,-2, \ldots$ y considerando las fórmula (55), de (70) y (71) se tiene,

$$
\begin{array}{r}
\left\{\delta^{(k)}\left(P_{-}\right) * \delta^{(l)}\left(P_{-}\right)\right\}^{\wedge}= \\
\Gamma(k+l-n+3) \Gamma\left(\frac{n}{2}-k-1\right)\left(\pi^{\frac{n}{2}-1}\right)^{3}\left((-1)^{\frac{q-1}{2}}\right)^{3} \\
\Gamma\left(-\frac{n}{2}+k+l+2\right) \Gamma\left(\frac{n}{2}-l-1\right)\left\{P_{+}{ }^{\frac{n}{2}-k-l-2}\right\}^{\wedge}
\end{array}
$$

si $q$ es impar y $p$ es par ( $n$ impar ) y $k+l+2>n-1$,

$$
\begin{array}{r}
\left\{\delta^{(k)}\left(P_{+}\right) * \delta^{(l)}\left(P_{+}\right)\right\}^{\wedge}=-\Gamma(k+l-n+3) \Gamma\left(\frac{n}{2}-k-1\right)\left(\pi^{\frac{n}{2}-1}\right)^{3}\left((-1)^{\frac{q-1}{2}}\right)^{3} \\
\Gamma\left(-\frac{n}{2}+k+l+2\right) \Gamma\left(\frac{n}{2}-l-1\right)\left\{P_{+}^{\frac{n}{2}-k-l-2}\right\}^{\wedge}
\end{array}
$$

si $q$ es impary $p$ es par ( $n$ impar) y $k+l+2>n-1$,

$$
\begin{array}{r}
\left\{\delta^{(k)}\left(P_{-}\right) * \delta^{(l)}\left(P_{-}\right)\right\}^{\wedge}=2^{2 n-2(k+1+l+1)}\left(\pi^{\frac{n}{2}}\right)^{2}\left((-1)^{\frac{q}{2}}\right)^{2} . \\
\delta^{\left(\frac{n}{2}-k-1\right)}\left(Q_{-}\right) . \delta^{\left(\frac{n}{2}-l-1\right)}\left(Q_{-}\right)
\end{array}
$$

si $p$ y $q$ son pares y $k<\frac{n}{2}-1$ y $l<\frac{n}{2}-1$, 


$$
\begin{array}{r}
\left\{\delta^{(k)}\left(P_{+}\right) * \delta^{(l)}\left(P_{+}\right)\right\}^{\wedge}=2^{2 n-2(k+1+l+1)}\left(\pi^{\frac{n}{2}}\right)^{2}\left((-1)^{\frac{p}{2}}\right)^{2} . \\
\delta^{\left(\frac{n}{2}-k-1\right)}\left(Q_{+}\right) \cdot \delta^{\left(\frac{n}{2}-l-1\right)}\left(Q_{+}\right)
\end{array}
$$

si $p$ y $q$ son pares y $k<\frac{n}{2}-1$ y $l<\frac{n}{2}-1$.

De (68) y (69) se tiene,

$$
\begin{aligned}
\left\{\delta^{(k)}\left(P_{+}\right) * \delta^{(l)}\left(P_{+}\right)\right\}^{\wedge}= & (-1)^{k+l+q-1} 2^{2 n-2(k+1+l+1)-2}\left(\pi^{\frac{n}{2}}\right)^{2} \\
& \Gamma\left(\frac{n}{2}-k-1\right) \Gamma\left(\frac{n}{2}-l-1\right) p f Q^{k+l-n+2}
\end{aligned}
$$

si $p$ y $q$ son impares y $k<\frac{n}{2}-1$ y $l<\frac{n}{2}-1$, y

$$
\begin{aligned}
\left\{\delta^{(k)}\left(P_{-}\right) * \delta^{(l)}\left(P_{-}\right)\right\}^{\wedge}= & 2^{2 n-2(k+1+l+1)-2}\left(\pi^{\frac{n}{2}}\right)^{2}(-1)^{q-1} \\
& \Gamma\left(\frac{n}{2}-k-1\right) \Gamma\left(\frac{n}{2}-l-1\right) p f Q^{k+l-n+2}
\end{aligned}
$$

si $p$ y $q$ son impares y $k<\frac{n}{2}-1_{\text {y }} l<\frac{n}{2}-1$.

Por otra parte, usando la fórmula

$$
\delta^{(k)}(P) \cdot \delta^{(l)}(P)=0
$$

cuando $n$ es par y $k+l+2 \neq \frac{n}{2}, \frac{n}{2}+1, \ldots$ ([6], página 18, fórmula 42), se tiene

$$
\delta^{\left(\frac{n}{2}-k-2\right)}\left(P_{+}\right) \cdot \delta^{\left(\frac{n}{2}-l-2\right)}\left(P_{+}\right)=0
$$

$\mathrm{y}$

$$
\delta^{\left(\frac{n}{2}-k-2\right)}\left(P_{-}\right) \cdot \delta^{\left(\frac{n}{2}-l-2\right)}\left(P_{-}\right)=0
$$

si $n$ es par y $k+l \neq \frac{n}{2}, \frac{n}{2}-1, \frac{n}{2}-2 \ldots$

Ahora usando la fórmula (67) se tiene

$$
p f Q^{-(n-k-l-2)}=2^{-1}\left[(Q+i 0)^{-(n-k-l-2)}+(Q-i 0)^{-(n-k-l-2)}\right] .
$$

Por otro parte, usando la fórmula (48), se tiene,

$$
\begin{gathered}
\left\{P^{\frac{n}{2}-k-l-2}\right\}^{\wedge}=-2^{n+2\left(\frac{n}{2}-k-l-2\right)-1} \pi^{\frac{n}{2}-1} \Gamma\left(\frac{n}{2}-k-l-2\right) \Gamma(n-k-l-2) \\
(-1)^{\frac{q-1}{2}}\left[(Q+i 0)^{-(n-k-l-2)}+(Q-i 0)^{-(n-k-l-2)}\right]
\end{gathered}
$$




\section{A. Aguirre}

Si $p$ y $q$ son impares.

De (77) y (78) y usando las fórmulas (82) y (83) se tiene

$$
\begin{aligned}
\left\{\delta^{(k)}\left(P_{-}\right) * \delta^{(l)}\left(P_{-}\right)\right\}^{\wedge}= & \pi^{\frac{n}{2}+1} 2^{-2} \Gamma\left(\frac{n}{2}-k-1\right) \Gamma\left(\frac{n}{2}-l-1\right) . \\
& \frac{1}{(-1)^{\frac{q-1}{2}} \Gamma\left(\frac{n}{2}-k-l-1\right) \Gamma(n-k-l-2)}\left\{P^{\frac{n}{2}-k-l-2}\right\}^{\wedge}
\end{aligned}
$$

si $p$ y $q$ son impares y

$$
\begin{aligned}
& \left\{\delta^{(k)}\left(P_{+}\right) * \delta^{(l)}\left(P_{+}\right)\right\}^{\wedge}=(-1)^{k+l} \pi^{\frac{n}{2}+1} 2^{-2} \Gamma\left(\frac{n}{2}-k-1\right) \Gamma\left(\frac{n}{2}-l-1 .\right. \\
& \frac{1}{(-1)^{\frac{q-1}{2}} \Gamma\left(\frac{n}{2}-k-l-1\right) \Gamma(n-k-l-2)}\left\{P^{\frac{n}{2}-k-l-2}\right\}^{\wedge}
\end{aligned}
$$

si $p$ y $q$ son impares.

De (73) y (74) y en virtud del teorema de la identidadde la transformada de Fourier, se llega a la formalización del siguiente teorema,

Teorema 1 Sean $k$ y $l$ enteros positivos y $\delta^{(k)}\left(P_{ \pm}\right)$las distribuciones definidas por (19) y (20) entonces si $k+l+2>n-1$ las siguientes fórmulas son válidas,

$$
\delta^{(k)}\left(P_{-}\right) * \delta^{(l)}\left(P_{-}\right)=g_{k, l} P_{+}^{\frac{n}{2}-k-l-2}
$$

si $q$ es impary $p$ es par ( $n$ impar ) y

$$
\delta^{(k)}\left(P_{+}\right) * \delta^{(l)}\left(P_{+}\right)=-g_{k, l} P_{+}^{\frac{n}{2}-k-l-2}
$$

si $q$ es impar y $p$ es par ( $n$ impar ). Donde $n$ es dimensión impar del espacio y

$$
\begin{gathered}
g_{k, l}=\quad \Gamma(k+l-n+3) \Gamma\left(\frac{n}{2}-k-1\right) \\
2^{n}\left(\pi^{\frac{n}{2}-1}\right)^{3}\left((-1)^{\frac{q-1}{2}}\right)^{3} \\
\Gamma\left(-\frac{n}{2}+k+l+2\right) \Gamma\left(\frac{n}{2}-l-1\right)
\end{gathered}
$$

observe que si $p$ es par y $q$ es impar, la distribución $P_{+}^{\lambda}$ es regular en $\lambda=-\frac{n}{2}-s, s=0,1,2, \ldots \quad$ ([1], página 260)

De (75), (76), usando (80), (81) y en virtud del teorema de identidad de la transformada de Fourier, se llega al siguiente teorema,

Teorema 2 Sean $k$ y $l$ enteros positivos y $\delta^{(k)}\left(P_{ \pm}\right)$distribuciones definidas por (21) y (22) entonces si $k<\frac{n}{2}-1$ y $l<\frac{n}{2}-1$ la siguiente fórmula es válida 


$$
\delta^{(k)}\left(P_{-}\right) * \delta^{(l)}\left(P_{-}\right)=0
$$

$y$

$$
\delta^{(k)}\left(P_{+}\right) * \delta^{(l)}\left(P_{+}\right)=0
$$

Si $p$ y $q$ son pares y $n=p+q$ es la dimensión del espacio.

Finalmente, de (84) y (85) y usando el teorema de identidad de la transformada de Fourier, se tiene el siguiente teorema,

Teorema 3 Sean $k$ y $l$ enteros positivos y $\delta^{(k)}\left(P_{ \pm}\right)$distribuciones definidas por (21) y (22) entonces si $k<\frac{n}{2}-1{ }_{y} l<\frac{n}{2}-1$ las siguientes fórmulas son válidas

$$
\delta^{(k)}\left(P_{-}\right) * \delta^{(l)}\left(P_{-}\right)=h_{k, l, n} P^{\frac{n}{2}-k-l-2}
$$

$y$

$$
\delta^{(k)}\left(P_{+}\right) * \delta^{(l)}\left(P_{+}\right)=(-1)^{k+l} h_{k, l, n} P^{\frac{n}{2}-k-l-2}
$$

Si $p$ y $q$ son impares $y n=p+q$ es la dimensión del espacio y

$$
h_{k, l, n}=\frac{\pi^{\frac{n}{2}+1} 2^{-2} \Gamma\left(\frac{n}{2}-k-1\right) \Gamma\left(\frac{n}{2}-l-1\right)}{\Gamma\left(\frac{n}{2}-k-l-1\right) \Gamma(n-k-l-2)(-1)^{\frac{q-1}{2}}}
$$

3. Los productos de convolución $\delta^{(k)}\left(P_{+}\right) * \delta^{(l)}\left(P_{+}\right), \delta^{(k)}\left(P_{-}\right) * \delta^{(l)}\left(P_{-}\right)$, $\delta_{1}^{(k)}(P) * \delta_{1}^{(l)}(P)$ y $\delta_{2}^{(k)}(P) * \delta_{2}^{(l)}(P)$.

Para obtener estos productos vamos a necesitar las siguientes fórmulas:

$$
\delta^{(k)}\left(P_{+}\right)=(-1) B_{k, p, q} L^{k-\frac{n}{2}+1} \text { si } k \geq \frac{n}{2}-1
$$

([2], página 10, fórmula 45 y 48),

$$
\delta^{(k)}\left(P_{-}\right)=2 B_{k, p, q} L^{k-\frac{n}{2}+1} \text { si } k \geq \frac{n}{2}-1
$$

([2], página 10, fórmula 46 y 49),

$$
\delta_{1}^{(k)}(P)=2 D_{k, p, q} L^{k-\frac{n}{2}+1}\{\delta(x)\} \text { para } p \text { y } q \text { par y } k \geq \frac{n}{2}-1
$$

([2], página 12, fórmula 54),

$$
\delta_{1}^{(k)}(P)=E_{k, p, q} L^{k-\frac{n}{2}+1}\{\delta(x)\} \text { para } p \text { y } q \text { impar y } k \geq \frac{n}{2}-1
$$

([2], página 11, fórmula 53),

$$
\delta_{2}^{(k)}(P)=D_{k, p, q} L^{k-\frac{n}{2}+1}\{\delta(x)\} \text { para } p \text { y } q \text { par y } k \geq \frac{n}{2}-1
$$

([2], página 12, fórmula 55) y

$$
\delta_{2}^{(k)}(P)=F_{k, p, q} L^{k-\frac{n}{2}+1}\{\delta(x)\} \text { para } p \text { y } q \text { impar y } k \geq \frac{n}{2}-1
$$


donde

$$
\begin{gathered}
B_{k, p, q}=\frac{(-1)^{k}(-1)^{\frac{q}{2}} \pi^{\frac{n}{2}}}{4^{k-\frac{n}{2}+1}\left(k-\frac{n}{2}+1\right) !} \text { para } p \text { y } q \text { pares } \\
B_{k, p, q}=\frac{(-1)^{k}(-1)^{\frac{q+1}{2}} \pi^{\frac{n}{2}-1}}{4^{k-\frac{n}{2}+1}\left(k-\frac{n}{2}+1\right) !}\left[\psi\left(\frac{p}{2}\right)-\psi\left(\frac{n}{2}\right)\right] L^{k-\frac{n}{2}+1}\{\delta(x)\} \\
\text { para } p \text { y } q \text { impares }
\end{gathered}
$$$$
D_{k, p, q}=-\frac{(-1)^{\frac{q}{2}} \pi^{\frac{n}{2}}}{4^{k-\frac{n}{2}+1}\left(k-\frac{n}{2}+1\right) !}
$$

para $p$ y $q$ pares,

$$
F_{k, p, q}=-\frac{(-1)^{\frac{q+1}{2}} \pi^{\frac{n}{2}-1}}{4^{k-\frac{n}{2}+1}\left(k-\frac{n}{2}+1\right) !}\left[\psi\left(\frac{p}{2}\right)-\psi\left(\frac{n}{2}\right)\right] \cdot L^{k-\frac{n}{2}+1}\{\delta(x)\}
$$

y

$$
E_{k, p, q}=\frac{(-1)^{k}(-1)^{\frac{q+1}{2}} \pi^{\frac{n}{2}-1}}{4^{k-\frac{n}{2}+1}\left(k-\frac{n}{2}+1\right) !}\left\{2\left[\psi\left(\frac{n}{2}\right)-\psi\left(\frac{p}{2}\right)\right]\right\}
$$

para $p$ y $q$ impares.

Ahora vamos a considerar dos teoremas para expresar los resultados, lo cual se hará a través de dos casos, cuando $\quad p$ y $q$ son pares y cuando $p$ y $q$ son impares.

Teorema 4 Sean $k, l$ enteros no negativos tales que $k \geq \frac{n}{2}-1$ y $l \geq \frac{n}{2}-1$ entonces si $p$ y $q$ son pares las siguientes fórmulas son válidas,

$$
\begin{gathered}
\delta^{(k)}\left(P_{+}\right) * \delta^{(l)}\left(P_{+}\right)=A_{k, l, n, p} \delta^{\left(k+l-\frac{n}{2}+1\right)}\left(P_{+}\right), \\
\delta^{(k)}\left(P_{-}\right) * \delta^{(l)}\left(P_{-}\right)=(-2) A_{k, l, n, p} \delta^{\left(k+l-\frac{n}{2}+1\right)}\left(P_{-}\right), \\
\delta_{1}^{(k)}(P) * \delta_{1}^{(l)}(P)=2 A_{k, l, n, q} \delta_{1}^{\left(k+l-\frac{n}{2}+1\right)}(P)
\end{gathered}
$$

$y$

$$
\delta_{2}^{(k)}(P) * \delta_{2}^{(l)}(P)=-A_{k, l, n, q} \delta_{2}^{\left(k+l-\frac{n}{2}+1\right)}(P)
$$

donde

$$
A_{k, l, n, j}=\frac{(-1)^{\frac{j}{2}}(k+l-n+2) !}{\left(k-\frac{n}{2}+1\right) !\left(l-\frac{n}{2}+1\right) !} \text { para } j=p o ́ q
$$

Prueba En la demostración del teorem 4, vamos a necesitar el producto de convolución del 


\section{A. Aguirre}

operador hiperbólico iterado $\left(j-\frac{n}{2}+1\right)$ veces $L^{k-\frac{n}{2}+1}\{\delta(x)\} * L^{l-\frac{n}{2}+1}\{\delta(x)\}$ resultado que aparece en ([2], página 346). Por lo tanto, de ( [2], página 346, fórmula 53), la siguiente fórmula es válida

$$
L^{k-\frac{n}{2}+1}\{\delta(x)\} * L^{l-\frac{n}{2}+1}\{\delta(x)\}=L^{k+l-\frac{n}{2}+1-\frac{n}{2}+1} \delta(x)
$$

bajo las condiciones $k \geq \frac{n}{2}-1, l \geq \frac{n}{2}-1$ para $n$ par.

Por otro lado sabemos que $L^{k-\frac{n}{2}+1}\{\delta(x)\}$ es una distribución de la clase $O_{c}^{\prime}$, donde $O_{c}^{\prime}$ es el espacio de distribución que se disminuye rápidamente ([7], página 244),Por lo tanto tomando en cuentas las fórmulas (91), (92), (93), (94), (95) y (96) los productos de convolución $\delta^{(k)}\left(P_{+}\right) * \delta^{(l)}\left(P_{+}\right), \delta^{(k)}\left(P_{-}\right) * \delta^{(l)}\left(P_{-}\right), \delta_{1}^{(k)}(P) * \delta_{1}^{(l)}(P)$ y $\delta_{2}^{(k)}(P) * \delta_{2}^{(l)}(P)$ existe bajo condiciones $k \geq \frac{n}{2}-1, l \geq \frac{n}{2}-1$ por $n$ par.

Por tanto, se tiene

$$
\begin{gathered}
\delta^{(k)}\left(P_{+}\right) * \delta^{(l)}\left(P_{+}\right)=B_{k, p, q} B_{l, p, q}\left(L^{k-\frac{n}{2}+1}\{\delta(x)\} * L^{l-\frac{n}{2}+1}\{\delta(x)\}\right) \\
\delta^{(k)}\left(P_{+}\right) * \delta^{(l)}\left(P_{+}\right)=4 B_{k, p, q} B_{l, p, q}\left(L^{k-\frac{n}{2}+1}\{\delta(x)\} * L^{l-\frac{n}{2}+1}\{\delta(x)\}\right)
\end{gathered}
$$

donde $B_{k, p, q}$ está definida por la fórmula (100) si $p$ y $q$ son pares y (101) si $p$ y $q$ son impares,

$$
\delta_{1}^{(k)}(P) * \delta_{1}^{(l)}(P)=4 D_{k, p, q} D_{l, p, q}\left(L^{k-\frac{n}{2}+1}\{\delta(x)\} * L^{l-\frac{n}{2}+1}\{\delta(x)\}\right)
$$

donde $D_{k, p, q}$ está definido por la fórmula (102),

$$
\delta_{1}^{(k)}(P) * \delta_{1}^{(l)}(P)=E_{k, p, q} E_{l, p, q}\left(L^{k-\frac{n}{2}+1}\{\delta(x)\} * L^{l-\frac{n}{2}+1}\{\delta(x)\}\right)
$$

donde $E_{k, p, q}$ está definido por la fórmula (104),

$$
\delta_{2}^{(k)}(P) * \delta_{2}^{(l)}(P)=D_{k, p, q} D_{l, p, q}\left(L^{k-\frac{n}{2}+1}\{\delta(x)\} * L^{l-\frac{n}{2}+1}\{\delta(x)\}\right)
$$

donde $D_{k, p, q}$ está definido por la fórmula (102) y

$$
\delta_{2}^{(k)}(P) * \delta_{2}^{(l)}(P)=F_{k, p, q} F_{l, p, q}\left(L^{k-\frac{n}{2}+1}\{\delta(x)\} * L^{l-\frac{n}{2}+1}\{\delta(x)\}\right)
$$

donde $E_{k, p, q}$ está definido por la fórmula (form.102) y

De (111), usando (110) y la fórmula (100) se obtiene la fórmula (105).

En forma similar de (112), usando (110) y la fórmula (100) se obtiene la fórmula (106).

De (113), usando (110) y la fórmula (102) se obtiene la fórmula ((107).

Finalmente de (115),usando(110)y la fórmula (form.102) se obtiene la fórmula (108).

Teorema 5 Sean $k, l$ enteros no negativos tales que $k \geq \frac{n}{2}-1$ y $l \geq \frac{n}{2}-1$ entonces si $p$ y $q$ son impares las siguientes fórmulas son válidas,

$$
\delta^{(k)}\left(P_{+}\right) * \delta^{(l)}\left(P_{+}\right)=C_{k, l, n, p} \delta^{\left(k+l-\frac{n}{2}+1\right)}\left(P_{+}\right)
$$




$$
\begin{gathered}
\delta^{(k)}\left(P_{-}\right) * \delta^{(l)}\left(P_{-}\right)=2 C_{k, l, n, p} \delta^{\left(k+l-\frac{n}{2}+1\right)}\left(P_{-}\right) \\
\delta_{1}^{(k)}(P) * \delta_{1}^{(l)}(P)=H_{k, l, n, p} \delta_{1}^{\left(k+l-\frac{n}{2}+1\right)}(P)
\end{gathered}
$$

$y$

$$
\delta_{2}^{(k)}(P) * \delta_{2}^{(l)}(P)=C_{k, l, n, q} \delta_{2}^{\left(k+l-\frac{n}{2}+1\right)}(P)
$$

donde

$$
C_{k, l, n, j}=\frac{(-1)\left(-\frac{1}{)^{\frac{j+1}{2}}}(k+l-n+2) !\right.}{\left(k-\frac{n}{2}+1\right) !\left(l-\frac{n}{2}+1\right) !} \cdot\left[\psi\left(\frac{j}{2}\right)-\psi\left(\frac{n}{2}\right)\right] \text { para } j=p o ́ q
$$

$y$

$$
H_{k, l, n, p}=\frac{(-1)^{\frac{p+1}{2}} \pi^{\frac{n}{2}-1}}{\left(k-\frac{n}{2}+1\right) !\left(1-\frac{n}{2}+1\right) !} \cdot\left\{\left[\psi\left(\frac{n}{2}\right)-\psi\left(\frac{p}{2}\right)\right]\right\}
$$

Prueba La demostración de este teorema5 se realiza de la misma forma que el teorema4. De (111), usando (110) y la fórmula (101) se obtiene la fórmula (117).

En forma similar de (112), usando (110) y la fórmula (101) se obtiene la fórmula (118). De (113), usando (110) y la fórmula (104) se obtiene la fórmula (form.119).

Finalmente de (116), usando (110) y la fórmula (103) se obtiene la fórmula (120).

\section{REFERENCIAS}

[1]. M. Gelfand and G.E. Shilov., Generalized Functions, Vol.I, Academic Press, New York,1964.

[2]. M. A. Aguirre T., Proportonality of $k$-th derivative of Dirac delta in hypercone, Mathematica Balkanica, New Series, vol.14, 2000, Fasc.3-4, pp.253-264.

[3]. M. A. Aguirre T., The distribution $\delta^{(k)}\left(P \pm i 0-m^{2}\right)$, Journal of Computational and Applied Mathematics, vol.88, 339-348, 1997.

[4]. M. A. Aguirre T., The expansion and Fourier's Transform of $\delta^{(k-1)}\left(m^{2}+P\right)$, Integral Transform and Special Functions, Vol.3, Nro.2, pp.113-134,1995.

[5]. M. A. Aguirre T., The product of convolution $P_{ \pm}^{\lambda} * P_{ \pm}^{\mu}$ and the multiplicative product $P_{ \pm}^{\lambda} . \delta^{(k)}\left(P_{ \pm}\right)$, Mathl.Comput.Modeling Vol.23, Nro.10, pp.135-144,1996.

[6]. M. A. Aguirre T., The distributional product of Dirac's delta in a hypercone, Journal of Computational and Applied Mathematics 115,pp13-21,(2000).

[7]. $\quad$ L. S. Schwartz., Theorie des Distributions, Hermann, Paris,1966.

A. Erdelyi (Editor)., Higher Trascendental Functions, Vol.I and II, McGraw-Hill, New York, 1953.

[8]. D.W.Bresters, On distributions connected with quadratic forms, SIAM, J.Appl. Math.,16, 1968, pp. 563-581.

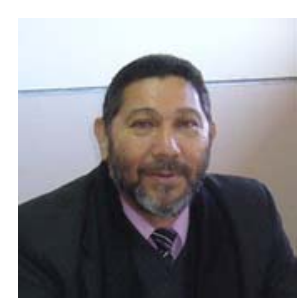

Manuel A. Aguirre, Profesor y Decano

Facultad de Ciencias Exactas

Universidad Nacional del Centro de la Prov. de Buenos Aires

Paraje Arroyo Seco, 7000-Tandil

Provincia de Buenos Aires, Argentina

Tel.: +54 2293439657

E-mail: maguirre@exa.unicen.edu.ar 